The Astrophysical Journal SUPPlement Series, 90:889-892, 1994 February

(c) 1994. The American Astronomical Society. All rights reserved. Printed in U.S.A.

\title{
ELECTRON ACCELERATION IN SHORT-LIVED RADIO TRANSIENTS
}

\author{
LEWIS BALL \\ Research Centre for Theoretical Astrophysics, University of Sydney, NSW 2006 Australia; e-mail: ball@physics.su.oz.au (Internet) \\ Received 1993 February 16; accepted 1993 April 30
}

\begin{abstract}
A short-lived (weeks-months) burst of radio emission, assumed to be synchrotron radiation, is sometimes associated with objects detected as soft X-ray transients. The "synchrotron bubble model" which has been applied to these radio transients assumes that the source is initially optically thick, and the model therefore takes no account of the details of the electron acceleration required. The initial increase in the radio flux is attributed to decreasing absorption in the expanding source. Observations of the soft X-ray transient GRS $1124-68$ (X-ray Nova Muscae 1991 ) showed, for the first time, the rising phase of such a radio transient. Another radio source, the Galactic Center Transient, was discovered serendipitously in the radio and observed throughout its rise and decay. In both cases the radio flux was a decreasing function of frequency throughout the periods of increasing and decreasing flux, which is not consistent with the optically thick assumption of the synchrotron bubble model. When this assumption is discarded the mechanism responsible for producing the ultrarelativistic electrons, or at the very least the rate at which those electrons become available, must be considered. A generalization of the simple model to include a source which supplies relativistic electrons with a constant energy spectrum is discussed. While such a model can go some way toward matching the observations, it is suggested that the time is ripe for an explicit model of the electron acceleration mechanism. Some of the features such a model may have are presented.
\end{abstract}

Subject headings: acceleration of particles - radio continuum: general — radiation mechanisms: nonthermal - X-rays: bursts

\section{INTRODUCTION}

Radio emission is sometimes detected from sources which are discovered as soft X-ray transients by all-sky X-ray cameras. Such objects are generally not sources of repeated outbursts of emission, or if they are, then the periods are very long, and so they are very rarely first discovered in the radio regime because of the very small field of view of modern radio telescopes. The strongest soft X-ray transients are generally looked for at wavelengths outside the $X$-ray regime once a sufficiently accurate position has been obtained, and at least one soft X-ray transient has been observed in the radio, infrared, optical, ultraviolet, $\mathrm{X}$-ray, and gamma-ray regimes. It is the radio emission from such objects which is of primary concern here. This emission can only be studied for a relatively short time after detection, generally a period of weeks or months, and it is particularly difficult to obtain observations of the onset phases of the outbursts.

It is generally accepted that soft $\mathrm{X}$-ray transients are associated with low-mass binary systems, consisting of a low-mass main-sequence star and a compact object which may be either a neutron star or a black hole (see, e.g., van Paradijs \& Verbunt 1984). Models for the transient X-ray emission are still the subject of some debate, but there is agreement that it is almost certainly due to a rapid increase in the rate at which matter is transferred from the main-sequence star to its compact companion. The models can be divided into two broad categories according to the site at which the increase in the rate of matter transfer occurs (van Paradijs \& Verbunt 1984). The mass transfer instability models involve a jump in the rate at which matter is transferred from the main-sequence star to the accre- tion disk surrounding the compact object, thickening and heating the disk. The disk instability models involve a rapid increase in the rate of matter transfer from the accretion disk onto the compact object, leading to a thinning of the disk. In both cases, gravitational potential energy will be converted into thermal energy, and it is believed that the resultant rapid heating of material produces the X-ray outburst.

Some of the soft X-ray transients which have been identified in the radio are A0620-00, Cen X-4, ASM 2000+25, V404 Cyg (GS 2023+338), and GRS 1124-68 (X-ray Nova Muscae 1991) (Hjellming et al. 1988; Han \& Hjellming 1990, 1992; Ball et al. 1993). It is interesting to note that this group contains what are probably the strongest stellar black hole candidates known to date, namely, A0620-00 and GRS 1124-68 (McClintock \& Remillard 1986; Della-Valle, Jarvis, \& West 1991a; Bailyn 1992; Goldwurm et al. 1992; Sunyaev et al. 1992; Remillard, McClintock, \& Bailyn 1993), and this is just one of many reasons soft X-ray transients have become the subject of intense interest in recent years. Although the radio emission from these objects is energetically unimportant, with far more energy being radiated in X-rays, the radio emission is of particular interest since it has the potential to provide insight into such things as the distribution of material and the magnetic field morphology in the binary systems. However, at this stage the processes responsible for the observed radio emission are very poorly understood. The nonthermal frequency spectrum of the radio emission has led to it being interpreted as synchrotron emission from ultrarelativistic electrons even though the other characteristic of synchrotron emission, very high degrees of linear polarization, has not been observed. Beyond this, it has been suggested that the unstable accretion 
event that leads to the soft X-ray transient outburst may produce a shock wave. Shock acceleration of electrons is apparently a very efficient process, and it is thought to be responsible for synchrotron emission in a variety of astrophysical sources (e.g., Blandford \& Eichler 1987; Jones \& Ellison 1991). So the production of a shock by the accretion event could provide a direct link between the observed X-ray and radio outbursts (Hjellming et al. 1988), although at this stage there is little solid evidence for this.

\section{OBSERVATIONS AND MODELING OF RADIO TRANSIENTS}

Observations of most short-lived radio transients have been hampered by the "detection delay"-the delay between the detection of the soft X-ray transient and the start of radio observations. This delay, typically a couple of weeks or more, has meant that in all but two recent transients the radio flux had already started decreasing when radio observations began. So with the exception of these two transients, the rising phase of the radio emission from these objects was not observed. The general features observed in the radio emission from A0620-00, Cen X-4, and ASM 2000+25 (Hjellming et al. 1988), and also from V404 Cyg (GS 2023+338) (Han \& Hjellming 1990, 1992), were as follows. The decay phase is typically quite rapid, with the flux at each frequency decreasing as a power law in time, and the frequency spectrum at each time also falling as a power law, so that $S \propto \nu^{-\alpha} t^{-\zeta}$ where $S$ is the flux density and $\alpha$ and $\zeta$ are positive constants. Typical values of $\alpha$ are in the range $0.5-1$. The value of $\zeta$ is not well determined because of the ambiguity in choosing a start time at which $t=0$. Han \& Hjellming (1992) report that the emission from V404 Cyg also displayed an extended period of much slower decay after the initial rapid decay, but we will not address such features here.

The observed radio emission from $\mathrm{A} 0620-00$, Cen X-4, and ASM 2000+25 (Hjellming et al. 1988) and the initial decay phase of V404 Cyg (Han \& Hjellming 1990, 1992) has been modeled using the synchrotron bubble model which is essentially due to van der Laan (1966). This model consists of an adiabatically expanding, spherically symmetric bubble of ultrarelativistic electrons and magnetic field. The magnetic field and the electron number density are assumed to be uniform throughout the bubble at all times. The magnetic field is taken to vary as $B \propto r^{-2}$, where $r$ is the radius of the bubble, and the condition for adiabatic expansion is that the electron energies vary as $E \propto r^{-1}$. The ultrarelativistic electrons are assumed to have a power-law energy distribution of the form $N(E) \propto E^{-\gamma}$ where $\gamma$ is a positive constant. Hjellming \& Johnston (1988) included the effect of the variation in the length of the line of sight through the spherical source.

In applying the synchrotron bubble model the electron acceleration process is ignored. It is assumed that the bubble starts out optically thick and that the electron acceleration process ceases before the radio emission can escape from the bubble. Thus the number of electrons in the bubble is a constant, fixed by the initial conditions. The increase in the radio flux from the source then comes about as the decreasing electron number density resulting from the expansion of the bubble leads to a decrease in the synchrotron self-absorption in the bubble. Eventually, when the effective optical depth through the bub- ble is much less than 1, the adiabatic energy losses of the electrons begin to dominate and the radio flux peaks and then decreases with time. So the synchrotron bubble model features two simple phases of emission. First, there is an optically thick rising phase in which, for a bubble expanding at a constant speed (Hjellming \& Johnston 1988; Hjellming et al. 1988; Ball $\&$ Vlassis 1993), the flux density $S$ varies as

$$
S \propto t^{3} \nu^{5 / 2},
$$

where the time $t$ is the apparent age of the bubble implied by its (constant) expansion rate and its radius at a given epoch. (The proportionality of $S$ to $t^{3}$, and thus to the emitting volume, is an artifact due to the assumption $B \propto r^{-2}$ rather than a general result.) The frequency spectrum during this phase, which is proportional to $\nu^{5 / 2}$, is typical of a self-absorbed synchrotron source. The radio flux then peaks when the effective optical depth of the bubble is of order one. The frequency spectrum changes its character around this emission peak, and an optically thin decay phase follows, in which

$$
S \propto t^{-2 \gamma} \nu^{-(\gamma-1) / 2},
$$

where $\gamma$ is the power-law index of the electron energy distribution. The qualitative features of this power-law decay are precisely those observed from short-lived radio transients, and fits of the model to the data are quite good (Hjellming et al. 1988; Han \& Hjellming 1990, 1992). However, the model fits are poorly constrained and somewhat unconvincing because of the lack of information regarding the actual epoch of the bursts and the lack of observations of the rising phases of the radio emission (Ball \& Vlassis 1993; Vlassis 1992; Ball et al. 1993).

\section{TWO RECENT RADIO TRANSIENTS}

Observations of two recent radio transients, GRS 1124-68 (X-ray Nova Muscae 1991) and the Galactic Center Transient, have added considerably to our knowledge of such objects.

GRS $1124-68$ is particularly notable for the wealth of data collected over a very wide range of the frequency spectrum, from radio wavelengths to gamma-ray energies (Della-Valle, Jarvis, \& West 1991a,b; Kesteven \& Turtle 1991; Brandt et al. 1992; Kitamoto et al. 1992; Kitamoto 1993). GRS 1124-68 was first detected on 1991 January 8 as a soft X-ray transient (Brandt et al. 1992). Radio observations began some 9 days later (Kesteven \& Turtle 1991), and two major bursts of radio emission were detected. The first burst (the January 17 Event) was seen only in decay, it having already peaked by the time observations began. The decay of the January 17 Event can be reasonably well fitted by a synchrotron bubble model with an electron energy distribution of power-law index $\gamma \approx 2.2$, assuming an apparent initial epoch of January 15 (Ball et al. 1993). Of more importance is the second radio burst (the January 30 Event), observed at $843 \mathrm{MHz}$ with the Molonglo Observatory Synthesis Telescope and at $4.8 \mathrm{GHz}$ with the Australia Telescope Compact Array. Brandt et al. (1992) noted that the interval between this burst and the first observed radio burst was $\sim 13$ days, and that there were two major $\mathrm{X}$-ray bursts observed from this source which were also $\sim 13$ days 
apart. This observation, tenuous as it is, is possibly the first evidence for a direct causal connection between the X-ray and the radio emission from these systems. The January 30 Event was observed during both the rising and the decay phases, and the flux at $843 \mathrm{MHz}$ exceeded that at $4.8 \mathrm{GHz}$ throughout the event (Kesteven \& Turtle 1991; Ball et al. 1993). This is the first time that a radio burst from such a transient has been observed during the rising phase, and the observed frequency dependence is inconsistent with the optically thick self-absorbed spectrum of the rising phase of the synchrotron bubble model, in which the flux is an increasing function of frequency.

The Galactic Center Transient was discovered serendipitously during radio observations of the Galactic center region (Zhao et al. 1992). In this case there was no "detection delay," and because of the observing schedule multifrequency data were obtained throughout the entire radio event, including the rising phase. Very little is known about this object in other frequency regimes, partly because of the extinction toward the Galactic center due to intervening dust. In particular, there is no X-ray information available, but on the basis of the radio data and limits on the infra-red emission from the object, Zhao et al. (1992) concluded that it could well be a similar type of object to the radio transients associated with soft X-ray transients. However, the observed radio spectrum of the Galactic Center Transient was a decreasing function of frequency throughout the event, with a spectral index of $\alpha \sim 1$, where $S \propto \nu^{-\alpha}$. This again is inconsistent with the optically thick rising phase of the synchrotron bubble model.

\section{DISCUSSION}

Since the observations of GRS 1124-68 and the Galactic Center Transient are inconsistent with the synchrotron bubble model which had been applied to earlier radio transients, there is clearly a need for a modified or alternative model. Furthermore, since the observations show that the emission is optically thin during the rising phase, detailed observations of the rising phases of these and other transients should reveal information regarding the process responsible for the acceleration of the electrons which produce the synchrotron emission. In turn, this may provide clues to the source morphology, including information regarding the shock at which the electron acceleration is assumed to occur.

A first step in modifying the synchrotron bubble model has been taken by Vlassis (1992) who included the effect of timedependent injection of ultrarelativistic electrons, relaxing the optically thick initial condition ( see also Ball \& Vlassis 1993). No attempt was made to model the acceleration process itself, it was simply assumed that the electrons were supplied to the bubble at a specified rate. The injected electrons were taken to have the same energy spectrum as those already in the bubble, so that $N(E, t)=f(t) E^{-\gamma}$, where $f(t)$ is a simple function of time. Marti, Paredes, \& Estalella (1992) used a very similar approach to include constant electron injection into a conical jet to model the variable radio emission from Cygnus X-3. The different geometries mean that the results reported by Vlassis (1992) and Ball \& Vlassis (1993) can not be directly compared with those of Marti et al. (1992). Vlassis (1992) found that the optically thin rises such as those observed from GRS 1124-68 and from the Galactic Center Transient could be obtained from a synchrotron bubble with electron injection such that the total number of electrons in the bubble, $N_{e}$, increased as $N_{e} \propto t^{3-6}$. Such injection rates are not infeasible if the acceleration occurs at an expanding shock front. For example, a spherical shock expanding at a constant speed into an electron distribution of constant number density, and accelerating a constant fraction of electrons encountered, will give $N_{e} \propto t^{3}$. This occurs because the shock area increases as $t^{2}$ and so the total number of accelerated electrons increases as $t^{3}$. More rapidly increasing injection rates could result from more extreme geometries.

Vlassis (1992) suggested that the transition of the radio transients from the rising phase to the decay phase could correspond to the cessation of the electron acceleration. The radio flux would increase while the acceleration process continues to supply ultrarelativistic electrons, but when that ceases the flux would decrease because of the adiabatic energy losses suffered by the electrons as the bubble continues to expand. Such a model is attractive since it retains the synchrotron bubble fits to the decay phases of the transients, while perhaps fitting the optically thin rising phases of GRS $1124-68$ and the Galactic Center Transient. Furthermore, it is particularly easy to imagine how such a "shut off" of the electron acceleration process may arise. Perhaps the most obvious would be that the shock wave simply expands beyond the region containing electrons to be accelerated. However, Vlassis (1992) was unable to obtain a self-consistent fit to the observations of the Galactic Center Transient with this piecemeal model. The problem which arises is that decay of a simple synchrotron bubble takes the form of equation (2), so the frequency spectral index $-(\gamma-1) / 2$ is related to the power-law index of the temporal decay $-2 \gamma$, assuming a constant rate of expansion and taking $t$ to be the apparent age of the bubble. The observed frequency spectrum implies that $\gamma \sim 2.2-3.4$ but the power-law index of the temporal decay, using the actual rather than the implied age given that the burst is known to have begun on around 1991 January 15, implies $\gamma \sim 0.5-1$. This discrepancy could be due to a slowing down in the expansion rate of the bubble. If the expansion rate decreases with time, then the apparent age of the bubble at any given time will exceed its actual age. The power-law index of the temporal decay implied from the synchrotron bubble fit will therefore exceed the power-law index obtained using the actual age. However, without a great deal more data than is available, little more can be said in this regard.

Irrespective of whether or not modifications to the synchrotron bubble model can be made which allow reasonable fits to the data, the model itself has a number of unsatisfactory features. Most significant of these are the assumptions of uniformity throughout the bubble. The assumption of randomly directed magnetic fields which remain uniform throughout the bubble and decrease as the square of the bubble radius, as the bubble expands, is very difficult to justify. Even more dubious is the assumption of a uniform number density of relativistic electrons in the bubble, particularly once injection of electrons into the source is included. Such electrons must surely be injected just downstream of the shock which is responsible for their acceleration. A more fruitful approach to the modeling of short-lived radio transients may therefore follow from a consideration of possible source morphologies with a view to develop- 
ing an appropriate time-dependent treatment of the acceleration of the electrons at an expanding shock. Such a model has been developed for the radio emission from SN 1987A (Ball \& Kirk 1992; Kirk, Duffy, \& Ball 1993). That model explicitly treats diffusive shock acceleration at an expanding spherical shock wave, and the result is an expression for the radio flux as a function of frequency and time.

Ball \& Kirk (1992) considered the case of a shock expanding at a constant speed into a magnetic field which varies as $1 / r$. Such a field is expected around a single magnetized star with an ionized wind, but may not be appropriate for the binary systems of interest here. Similarly, Ball \& Kirk assume that the electrons accelerated by the shock are picked up at a constant rate. This is appropriate for a spherical shock expanding at a constant speed into a medium where the density falls off as $1 / r^{2}$, as expected in a stellar wind, but again the applicability to the radio transients of interest here is uncertain. It may be that the radio source associated with short-lived radio transients is very large with respect to the size of the binary system, in which case the model of Ball \& Kirk (1992) may be directly applicable. Even if this is not true, a direct application of the Ball \& Kirk model is probably more realistic than the synchrotron bubble model. It gives an optically thin rise followed by an optically thin decay during which the flux obeys simple power laws in both frequency and time. It contains the only essential physics of the synchrotron bubble model, namely, synchrotron emission from a distribution of ultrarelativistic electrons which is undergoing adiabatic expansion, as well as addressing the crucial physics of the electron acceleration. Furthermore, generalization of the model of Ball \& Kirk is relatively straightforward. However, while the general structure of the region around SN 1987A where the radio emission is thought to originate is quite well known, the understanding of the overall geometry, magnetic field structure, and density profile in the sources of interest here is extremely limited. Although it is unlikely that this problem will be solved quickly, it is intriguing to speculate whether such a model applied to acceleration of electrons from the medium surrounding the binary system as a whole, from a halo around the accretion disk, or even from the disk itself, could explain the observed emission.

I thank Tony Turtle for first drawing the radio observations of GRS 1124-68 to my attention, and Mike Kesteven, Duncan Campbell-Wilson, and Bob Hjellming for subsequent discussions of those observations. I also thank Jun-Hui Zhao, Ravi Subrahmanyan and Miller Goss for many discussions regarding the Galactic Center Transient, and my Honours Year student Michael Vlassis for his work on generalizing the synchrotron bubble model.

\section{REFERENCES}

Bailyn, C. D. 1992, ApJ, 391, 298

Ball, L. T., Kesteven, M. J., Campbell-Wilson, D., Turtle, A. J., \& Hjellming, R. M. 1993, in preparation

Ball, L. T., \& Kirk, J. G. 1992, ApJ, 396, L39

Ball, L. T., \& Vlassis, M. 1993, Proc. Astron. Soc. Australia, in press Blandford, R. D., \& Eichler, D. 1987, Phys. Rep., 154, 1

Brandt, S., Castro-Tirado, A. J., Lund, N., Dremin, V., Lapshov, I., \& Sunyaev, R. 1992, A\&A, 254, L39

Della-Valle, M., Jarvis, B. J., \& West, R. M. 1991a, Nature, 353, 50 1991b, A\&A, 247, L33

Goldwurm, A.. et al. 1992, ApJ, 389, L79

Hjellming, R. M., Calovini, T. A., Han, X., \& Córdova, F. A. 1988, ApJ, $335, \mathrm{~L} 75$

Han, X., \& Hjellming, R. M. 1990, in Accretion-powered Compact Binaries, ed. C. Mauche (Cambridge Univ. Press), 25

. 1992, ApJ, 400, 304

Hjellming, R. M., \& Johnston, K. J. 1988, ApJ, 328, 600

Jones, F. C., \& Ellison, D. C. 1991, Space Sci. Rev., 58, 259
Kesteven, M. J., \& Turtle, A. J. 1991, in Proc. of the Workshop on Nova Muscae 1991, ed. N. Lund (Lyngby: Danish Space Res. Inst.), Lyngby, 115

Kirk, J. G., Duffy, P., \& Ball, L. T. 1993, ApJS, 89, 000

Kitamoto, S. 1993, in Proc. 4th Internat. Toki Conf. on Plasma Physics \& Controlled Nuclear Fusion (Toki, Japan), (ESA SP-351), 297

Kitamoto, S., Tsunemi, H., Miyamoto, S., \& Hayashida, K. 1992, ApJ, 394,609

Marti, J., Paredes, J. M., \& Estalella, R. 1992, A\&A, 258, 309

McClintock, J. E., \& Remillard, R. A. 1986, ApJ, 308, 110

Remillard, R., McClintock J., \& Bailyn, C. 1993, ApJ, 399, L145

Sunyaev, R., et al. 1992, ApJ, 389, L75

van der Laan, H. 1966, Nature, 211, 1131

van Paradijs, J., \& Verbunt, F. 1984, in AIP Conf. Proc., No. 115, HighEnergy Transients in Astrophysics, ed. S. Woosley (New York: AIP), 49

Vlassis, M. 1992, Synchrotron Bubbles and Radio Transients, Honours Rep., University of Sydney

Zhao, J., et al. 1992, Science, 255, 1538 